ARTICLE

https://doi.org/10.1038/s41467-019-12589-5

\title{
Structure of ribosome-bound azole-modified peptide phazolicin rationalizes its species-specific mode of bacterial translation inhibition
}

\author{
Dmitrii Y. Travin (10 1,2, Zoe L. Watson (10 ${ }^{3}$, Mikhail Metelev (1) 1,2,12, Fred R. Ward ${ }^{4}$, Ilya A. Osterman ${ }^{1,5}$, \\ Irina M. Khven ${ }^{5,6}$, Nelli F. Khabibullina (1) ${ }^{7,13}$, Marina Serebryakova (D) ${ }^{8}$, Peter Mergaert (1) ${ }^{9}$, \\ Yury S. Polikanov (i] ${ }^{7,10 \star}$, Jamie H.D. Cate (i] ${ }^{3,4 \star}$ \& Konstantin Severinov ${ }^{1,2,11 \star}$
}

Ribosome-synthesized post-translationally modified peptides (RiPPs) represent a rapidly expanding class of natural products with various biological activities. Linear azol(in)e-containing peptides (LAPs) comprise a subclass of RiPPs that display outstanding diversity of mechanisms of action while sharing common structural features. Here, we report the discovery of a new LAP biosynthetic gene cluster in the genome of Rhizobium Pop5, which encodes the precursor peptide and modification machinery of phazolicin ( $\mathrm{PHZ})$ - an extensively modified peptide exhibiting narrow-spectrum antibacterial activity against some symbiotic bacteria of leguminous plants. The cryo-EM structure of the Escherichia coli 70S-PHZ complex reveals that the drug interacts with the $23 \mathrm{~S}$ rRNA and uL4/uL22 proteins and obstructs ribosomal exit tunnel in a way that is distinct from other compounds. We show that the uL4 loop sequence determines the species-specificity of antibiotic action. PHZ expands the known diversity of LAPs and may be used in the future as biocontrol agent for agricultural needs.

\footnotetext{
${ }^{1}$ Center of Life Sciences, Skolkovo Institute of Science and Technology, Moscow, Russia. ${ }^{2}$ Institute of Gene Biology, Russian Academy of Science, Moscow, Russia. ${ }^{3}$ Department of Chemistry, University of California, Berkeley, USA. ${ }^{4}$ Department of Molecular and Cell Biology, University of California, Berkeley, USA. ${ }^{5}$ Department of Chemistry, Lomonosov Moscow State University, Moscow, Russia. ${ }^{6}$ Department of Bioengineering and Bioinformatics, Lomonosov Moscow State University, Moscow, Russia. ${ }^{7}$ Department of Biological Sciences, University of Illinois at Chicago, Chicago, IL, USA. ${ }^{8}$ A.N. Belozersky Institute of Physico-Chemical Biology, Lomonosov Moscow State University, Moscow, Russia. ${ }^{9}$ Institute for Integrative Biology of the Cell (I2BC), CNRS CEA University Paris-Sud, University Paris-Saclay, Gif-sur-Yvette, France. ${ }^{10}$ Department of Pharmaceutical Sciences, University of Illinois at Chicago, Chicago, USA. ${ }^{11}$ Waksman Institute for Microbiology, Rutgers, The State University of New Jersey, Piscataway, USA. ${ }^{12}$ Present address: Department of Cell and Molecular Biology, Uppsala University, Uppsala, Sweden. ${ }^{13}$ Present address: National Medical Research Center for Phthisiopulmology \& Infectious Diseases, Moscow, Russia. *email: yuryp@uic.edu; jcate@lbl.gov; severik@waksman.rutgers.edu
} 
S ince the discovery of the first antibiotics, natural products of bacterial and fungal origin serve as the predominant source of new compounds with various in-high-demand functional activities in the fight against ever-growing antibiotic resistance and cancer ${ }^{1,2}$. While traditional activity-based screens often lead to the rediscovery of already known compounds, the everincreasing genomic data make genome-mining-based strategies viable alternatives in the search for new bioactive compounds. Ribosomally synthesized and post-translationally modified peptides (RiPPs) comprise a diverse class of natural products that are well suited for genome-mining approaches as their biosynthetic gene clusters (BGCs) contain multiple signature genes coding for enzymes performing posttranslational modifications and the peptide precursor genes, allowing to significantly improve the quality of bioinformatics predictions ${ }^{3}$.

Linear azol(in)e-containing peptides (LAPs) form a subfamily of RiPPs sharing thiazol(in)e and (methyl)oxazol(in)e heterocycles, which result from cyclization of Cys and Ser (Thr) side chains during the posttranslational modification of the precursor peptide $^{4}$. In addition to the precursor peptide-coding gene (gene A) LAP BGCs typically include genes for an YcaO-domain containing cyclodehydratase (gene D), its partner protein (gene $\mathrm{C}$ or gene F), FMN-dependent dehydrogenase (gene B), which oxidizes azolines to azoles, and the efflux pump ${ }^{5}$. Although less than two dozens of LAPs can be considered as well-characterized, they demonstrate antibacterial activity through several completely different mechanisms. For example, microcin B17 is a DNAgyrase poison ${ }^{6}$, plantazolicin targets the membrane ${ }^{7}$, and klebsazolicin inhibits the ribosome $e^{8}$. This diversity of modes of action, unprecedented for peptides sharing common chemical features, makes LAPs a group of special interest for the search of new antibacterials.

Here, we report the discovery of a new LAP BGC in the genome of Rhizobium sp. Pop5, and characterization of its product, phazolicin (PHZ) - a previously unknown linear thiazole/oxazole-modified peptide, which targets the bacterial ribosome. The producing strain, a symbiotic nitrogen-fixing bacterium was isolated in a tropical forest in Los Tuxtlas (Mexico) from the root nodules of wild beans (Phaseolus vulgaris, hence "phazolicin"). Although the studies of natural products with antibacterial properties produced by rhizobia date back to the $1960 \mathrm{~s}^{9}$, the number of well-characterized compounds is still very small with trifolitoxin produced by R. leguminosarum bv. trifolii T24 being the only rhizobial thiazole/oxazole-containing RiPP described to date ${ }^{10}$. Being active against a variety of bacteria from Rhizobiales including several phytopathogens, such as Agrobacterium tumifaciens and A. rhizogenes, PHZ is not only interesting because of its idiosyncratic mode of interaction with the ribosome, but also as a potential agent for biocontrol in agriculture.

\section{Results}

Identification of a new LAP biosynthetic gene cluster. Klebsazolicin (KLB) from Klebsiella pneumoniae is a recently characterized LAP, which inhibits prokaryotic translation through the obstruction of the ribosome exit tunnel ${ }^{8}$. The BGC of this RiPP $(k l p A C B D E)$ contains genes encoding the precursor peptide $(k l p A)$, the three enzymes involved in the formation of azole cycles ( $k l p C, k l p B$, and $k l p D)$, as well as a transmembrane pump $(k l p E)$ exporting the modified peptide. We used the amino acid sequences of KlpB and KlpD proteins as queries to search the NCBI non-redundant protein database using the PSI-BLASTP algorithm ${ }^{11}$ followed by manual analysis of the genomic environment of the identified gene orthologues. Along with several previously described clusters of KLB homologs 8 a distinct BGC phzEACBD was found in the genome of Rhizobium sp. Pop5. Two other related BGCs were found in the genomes of Rhizobium sp. PDO1-076 and Phyllobacterium myrsinacearum DSM 5893, also belonging to the order Rhizobiales. The alignment of amino acid sequences of the predicted core parts of precursor peptides revealed a similar pattern of Cys/Ser/Thr residues, which potentially could be cyclized in the mature LAP product (Fig. 1b, red). The putative PhzA precursor peptide has an amino acid sequence significantly different from that of the KLB precursor KlpA (Fig. 1a). As in the case with KlpA, the C-terminal part of the predicted PhzA precursor peptide is enriched in Ser, Thr, and Cys residues but also contains several positively charged residues absent in the core part of KlpA. Moreover, PhzA and its relatives lack the $\mathrm{X}-\mathrm{X}-(\mathrm{S} / \mathrm{T}) \mathrm{P}$ motif required for the formation of the $\mathrm{N}$ terminal amidine cycle, which is strictly required for the activity of $\mathrm{KLB}^{12}$. Therefore, we hypothesized that the phzEACBD and similar BGCs from other Rhizobiales might encode closely related LAPs with structural and functional properties different from those of KLB or other known compounds of the family.

Purification and characterization of a new LAP. Reverse-phase HPLC analysis of medium after cultivation of the Rhizobium sp. Pop5 led to the identification of several fractions absorbing at 254 $\mathrm{nm}$, a wavelength previously used in the purification of other azole-containing compounds (Supplementary Fig. $1 \mathrm{~A})^{8,13}$. MALDI-TOF-MS analysis of the selected fractions showed the presence of compounds, which, based on the observed masses, appeared to be the products of the PhzA precursor peptide posttranslational modification (Supplementary Fig. 1B). The major compound with monoisotopic $\mathrm{MH}^{+} 2363.9$ was named PHZ (Supplementary Fig. 1C). This mass matches the mass of the C-terminal part of the PhzA precursor peptide, if it was cleaved between the two alanine residues [...Thr-Ala- $\downarrow$-Ala-Thr-Cys...] and contained eight azole rings (loss of $20 \mathrm{Da}$ per installation of each cycle corresponds to $-18 \mathrm{Da}$ for each dehydration/cyclization reaction and $-2 \mathrm{Da}$ for each azoline oxidation event) (Fig. 1c). Two other major compounds detected in the growth media (Supplementary Fig. 1B) also contained eight azole cycles but had an alternative site for the leader peptide cleavage situated one or two residues closer to the N-terminus of the precursor. These compounds are further referred as A-PHZ $\left(\mathrm{MH}^{+}=\right.$ 2434.9) and TA-PHZ $\left(\mathrm{MH}^{+}=2536.0\right)$. High-resolution MS measurements for PHZ, A-PHZ, and TA-PHZ resulted in MW values of $2362.8679,2433.9052$, and 2534.9524 , which are all within $1.5 \mathrm{ppm}$ of the corresponding masses calculated based on the brutto-formulas (Supplementary Fig. 2). Although due to extensive modification the fragmentation of the PHZ was relatively poor, MS/MS analysis using both ESI-MS/MS and MALDIMS/MS techniques allowed predictions of the positions of azole cycles in the structure of PHZ (Supplementary Fig. 3). PHZ is a 27-residue long peptide with three thiazole and five oxazole cycles in its structure. The cycles are distributed evenly across the core part of the precursor peptide with every third residue being turned into an azole (Fig. 1c). In addition to the major fractions, several minor forms with a fewer (5-7) number of azole cycles were detected in the cultivation medium (Supplementary Fig. 1B). Compounds with less than eight cycles lacked the C-terminal cycles, consistent with the $\mathrm{N}$ - to $\mathrm{C}$-terminus direction of modification of the $\mathrm{PHZ}$ precursor peptide.

PHZ demonstrates antimicrobial activity against Rhizobiales. When the purified PHZ was tested against Escherichia coli BW25113 grown on rich solid medium, small growth inhibition zones were observed only when the compound was applied in high concentrations (5-10 mM). Many known RiPPs demonstrate a narrow spectrum of antibacterial activity and are usually 
a

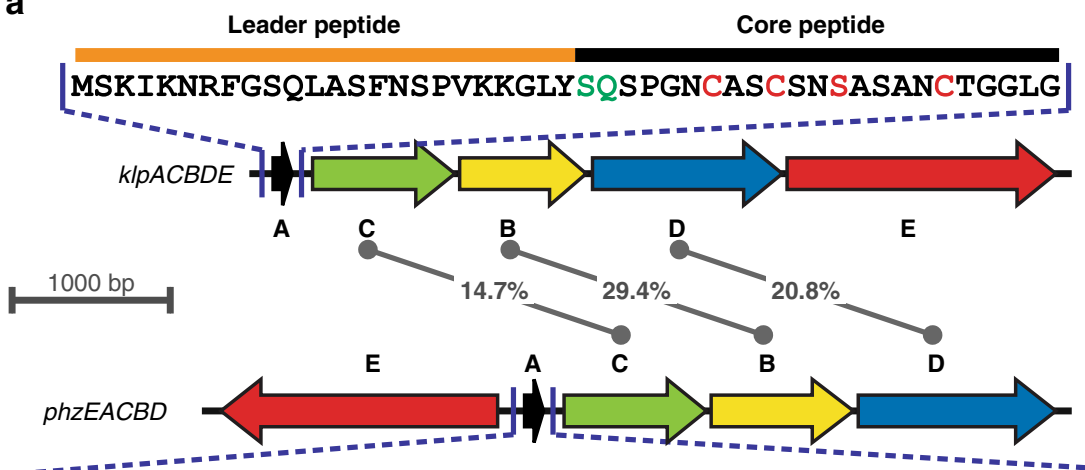

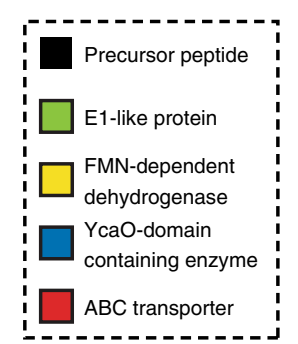

MTTQILNPPQFGTEIEFVDAGDSTVQTAATCARCDSSSRCGASGKSSGSASSIST

Predicted leader peptide

Predicted core peptide

b

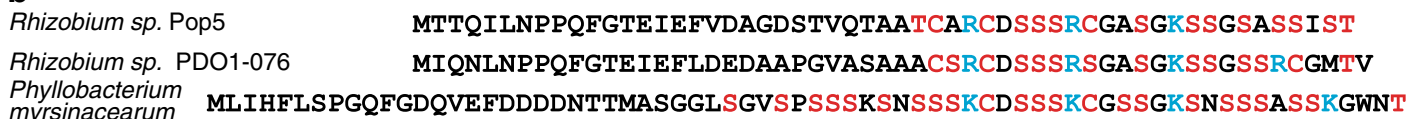

Phyllobacterium

C

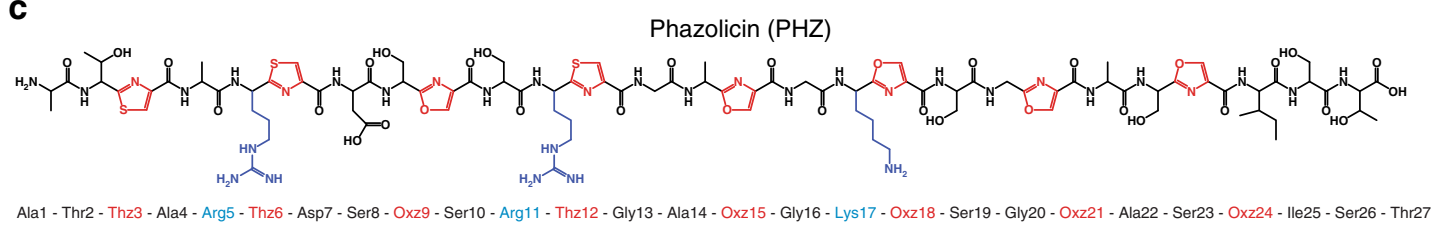

Fig. 1 Organization of the phazolicin (PHZ) biosynthetic gene cluster. a Comparison of klebsazolicin (KLB) biosynthetic gene cluster ( $k$ IpACBDE) with the cluster found in the genome of Rhizobium sp. Pop5. Functions of the genes forming the BGCs are listed on the right. The numbers in the middle indicate the extent of identity between the amino acid sequences of the $C, B$, and D gene products. The sequence of the KLB precursor peptide is shown above its BGC with the leader and core parts indicated. Residues converted to azoles in the final product are highlighted in red. Residues involved in the formation of the $\mathrm{N}$-terminal amidine cycle are highlighted in green. The sequence of the precursor peptide from the newly identified BGC is shown below it. Ser and Cys residues in the sequence of PhzA precursor involved in oxazole and thiazole formation, respectively, are shown in red. Positively charged residues of the predicted core part are highlighted in blue. $\mathbf{b}$ Alignment of the amino acid sequences of precursor peptides encoded in phzEACBD BGC and BGCs of PHZ homologs from other Rhizobiales (Rhizobium sp. PD01-076 and Phyllobacterium myrsinacearum DSM 5893). Ser, Thr, and Cys residues in the predicted core parts of the peptides are shown in red. Positively charged residues are highlighted in blue. c Structure of the fully modified PHZ peptide after the cleavage of the leader. Cyclized residues are shown in red (Thz and Oxz). Positively charged amino acids are highlighted in blue

active against bacteria, which are evolutionary close to the producing strain ${ }^{7,14,15}$ serving as powerful weapons in the competition for the ecological niche between closely related species. Accordingly, we tested PHZ against a panel of microorganisms from the order Rhizobiales (class Alphaproteobacteria) grown in a rich liquid medium. The compound proved to be highly active against bacteria from genera Rhizobium, Sinorhizobium (Ensifer), and Azorhizobium with the minimal inhibitory concentrations (MICs) being around $1 \mu \mathrm{M}$ (Table 1 ). PHZ is less active against two tested strains of Agrobacterium, while microorganisms from the genus Mesorhizobium appeared to be PHZ-resistant (Table 1). PHZ was also tested against a small set of plant pathogens, plantassociated and soil microorganisms from Gammaproteobacteria (Erwinia amylovora, Pantoea ananatis PA4, Pseudomonas putida KT2440, P. fluorescens Pf-5), Firmicutes (Bacillus subtilis 168, B. cereus ATCC 4342), and Actinobacteria (Arthrobacter sp. ATCC21022, Microtetraspora glauca NRRL B-3735). However, no activity was observed. Taken together, these observations demonstrate that, similarly to a number of other LAPs, PHZ is a narrow-spectrum antimicrobial compound specifically targeting several genera from Rhizobiales.

The resistance of producing strains to RiPPs with antimicrobial activity often results from the efficient export of the mature compound by a specific transporter encoded in the corresponding $\mathrm{BGC}^{16}$. However, there are examples of BGCs containing additional genes providing self-immunity through different mechanisms ${ }^{17,18}$. Expression of a plasmid-borne copy of the $p h z E$ gene, which was annotated as an exporter pump, in $S$. meliloti Sm1021 conferred resistance to external PHZ and increased the MIC more than 100-fold in comparison with the strain carrying an empty vector $(\lambda)$. These data confirm the role of PhzE transporter in self-immunity of the producing strain to PHZ.

PHZ inhibits bacterial protein translation in vivo and in vitro. To determine the mode of action of PHZ we tested its activity in vivo using an E. coli-based double-reporter system that allows one to identify antibiotics inducing ribosome stalling or inhibiting DNA replication ${ }^{19}$. In this system, pDualrep2 plasmid harbors two genes encoding fluorescent proteins under different regulation: the red fluorescent protein (RFP) gene is transcribed in the presence of sublethal concentrations of SOS-response inducing agents (e.g., fluoroquinolones or microcin B17), while the gene for far-red protein Katushka2S is transcribed only when translation inhibitors (e.g., macrolides) are present in the medium in sublethal concentrations. Thus, when a drop containing antimicrobial agent is deposited onto the lawn of cells carrying pDualrep2, the compound diffuses into the media and usually kills cells adjacent to the deposition spot. However, further away from the deposition spot at the outer edge of a growth inhibition zone the compound is present in a sublethal concentration and can induce production of either the RFP or Katushka2S 


\section{Table 1 Phazolicin MICs for various bacterial strains in rich} YEB medium and Rhizobium medium (RM)

\begin{tabular}{|c|c|c|c|}
\hline Microorganism (Strain) & Medium & $\begin{array}{l}\text { MIC } \\
(\mu \mathrm{M})\end{array}$ & $\begin{array}{l}\text { MIC } \\
(\mu \mathrm{g} / \mathrm{ml})\end{array}$ \\
\hline $\begin{array}{l}\text { Rhizobium leguminosarum bv. } \\
\text { viciae } 3841\end{array}$ & RM & 1 & 2.4 \\
\hline $\begin{array}{l}\text { Rhizobium leguminosarum bv. } \\
\text { phaseoli } 4292\end{array}$ & RM & 0.125 & 0.3 \\
\hline $\begin{array}{l}\text { Rhizobium leguminosarum bv. } \\
\text { phaseoli RCR } 3622\end{array}$ & RM & 2 & 4.7 \\
\hline Rhizobium etli DSM 11541 & RM & 0.25 & 0.6 \\
\hline Rhizobium tibethicum DSM 21102 & RM & 1 & 2.4 \\
\hline Agrobacterium tumefaciens C58C1 & YEB & 64 & 151.3 \\
\hline Agrobacterium rhizogenes ARQUA1 & YEB & 64 & 151.3 \\
\hline Mesorhizobium ciceri & YEB & $>128$ & $>302.6$ \\
\hline Mesorhizobum loti MAFF303099 & YEB & $>128$ & $>302.6$ \\
\hline $\begin{array}{l}\text { Mesorhizobium thianshanense } \\
\mathrm{HAMBI} 3372\end{array}$ & YEB & $>128$ & $>302.6$ \\
\hline Azorhizobium caulinodans ORS571 & YEB & 2 & 4.7 \\
\hline Sinorhizobium fredii $\mathrm{HH} 103$ & YEB & 0.25 & 0.6 \\
\hline Sinorhizobium medicae WSM419 & YEB & 4 & 9.5 \\
\hline Sinorhizobium meliloti Sm1021 & YEB & 1 & 2.4 \\
\hline Sinorhizobium meliloti Sm1021 pSRK & YEB & 1 & 2.4 \\
\hline $\begin{array}{l}\text { Sinorhizobium meliloti Sm1021 } \\
\text { pSRK-phzE }\end{array}$ & YEB & $>128$ & $>302.6$ \\
\hline
\end{tabular}

fluorescent protein depending on whether the compound targets DNA replication or translation, respectively. Similar to KLB and erythromycin (ERY), PHZ induces expression of Katushka2S but not RFP, indicating that $\mathrm{PHZ}$ is an inhibitor of translation (Fig. 2a, left panel). In this assay, we observed a difference in the size of PHZ-induced inhibition zones between the E. coli strain with the deleted tolC gene and the wild-type strain (Fig. 2a, right panel). TolC is a major outer membrane multidrug efflux porin that is responsible for the export of various compounds from the cell including siderophores (e.g., enterobactin ${ }^{20}$ ), macrolide antibiotics $^{21}$, and some RiPPs (e.g., microcin J25 ${ }^{22}$ ). In contrast to the situation observed with PHZ, the KLB-induced inhibition zones were comparable in size between the $\Delta$ tolC and the wildtype E. coli strains, suggesting that $\mathrm{PHZ}$ but not KLB is subject to TolC-dependent efflux.

To further confirm that PHZ is a protein synthesis inhibitor, we tested its ability to decrease the translation of the luciferase mRNA in vitro using an E. coli S30 extract. Concentrationdependent inhibition of in vitro translation by $\mathrm{PHZ}$ was observed (Fig. 2b) with $1 \mu \mathrm{M}$ of PHZ decreasing the luciferase signal by approximately three-fold (Fig. $2 \mathrm{~b}$, blue curve), while at $5 \mu \mathrm{M} \mathrm{PHZ}$ inhibited translation by $\sim 95 \%$ (Fig. 2b, red curve).

PHZ obstructs the nascent peptide exit tunnel (NPET) of the ribosome. Protein synthesis inhibitors can interfere with the activity of the ribosome or any of the various other enzymes associated with protein production (translation factors, aminoacyl-tRNA synthetases, etc.). Following the analogy with $\mathrm{KLB}$, we proposed that $\mathrm{PHZ}$ acts upon the bacterial ribosome. To determine the exact mechanism of translation inhibition by PHZ we attempted to crystallize the $70 \mathrm{~S}$ ribosome from Thermus thermophilus (Tth) in complex with PHZ, mRNA, and tRNAs, as previously done with $\mathrm{KLB}^{8}$, and several other translation inhibitors $^{23-30}$. Despite numerous attempts, we were not able to observe any positive electron density peaks corresponding to the ribosome-bound PHZ anywhere within the Tth 70S ribosome. Moreover, in vitro translation inhibition experiments using $E$. coli (Eco) $70 \mathrm{~S}$ or hybrid $70 \mathrm{~S}$ (Eco $30 \mathrm{~S}$ subunit + Tth $50 \mathrm{~S}$ subunit) demonstrated that $\mathrm{PHZ}$ does not target Tth ribosome a
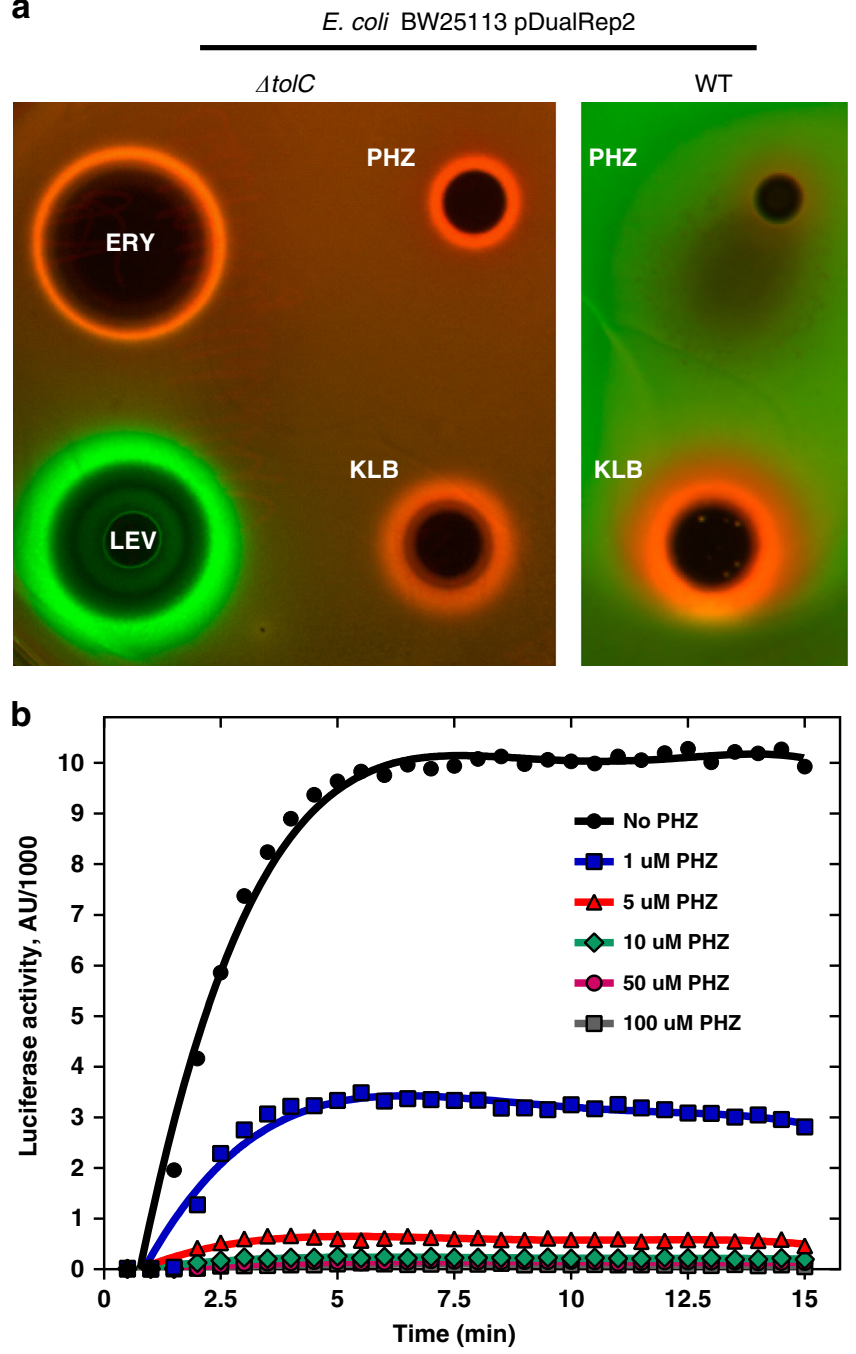

Fig. 2 Phazolicin is an inhibitor of protein synthesis both in vivo and in vitro. a In vivo testing of $\mathrm{PHZ}$ activity using BW25113 $\Delta$ tolC pDualRep2 and BW25113 pDualRep2 (WT) reporter strains. The induction of RFP expression (green halo around the inhibition zone, pseudocolors) is triggered by DNA-damage while the induction of Katushka2S protein (red halo) occurs in response to ribosome stalling. Erythromycin (ERY), levofloxacin (LEV), and klebsazolicin (KLB) are used as controls. b Kinetic curves showing inhibition of protein synthesis by increasing concentrations of $\mathrm{PHZ}$ in the in vitro cell-free translation in E. coli $\mathrm{S} 30$ extract. $\mathrm{AU}$ arbitrary units

(Supplementary Fig. 4). However, the same data also suggested that PHZ does target the large ribosomal subunit of the E. coli ribosome.

Since the crystallization-based approach was unsuccessful, we switched to cryo-EM to determine the structure of PHZ bound to the sensitive Eco $70 \mathrm{~S}$ ribosome. The obtained charge density map, characterized by the overall resolution of $2.87 \AA$ according to the "gold-standard" Fourier shell correlation (FSC) method (Supplementary Fig. 5A), revealed PHZ bound to the ribosome (Fig. 3a). The high resolution and excellent quality of the map in the PHZbinding site (Supplementary Fig. 5B), allowed us not only to fit its atomic model (residues 2-23; Fig. 3b) but also to confirm the positions of seven azoles in the structure of the modified peptide proposed according to the MS-MS analysis. While the results of tandem mass spectrometry indicate another oxazole present at the position 24, we left Ser23 unmodified in the model since Ser24 
a

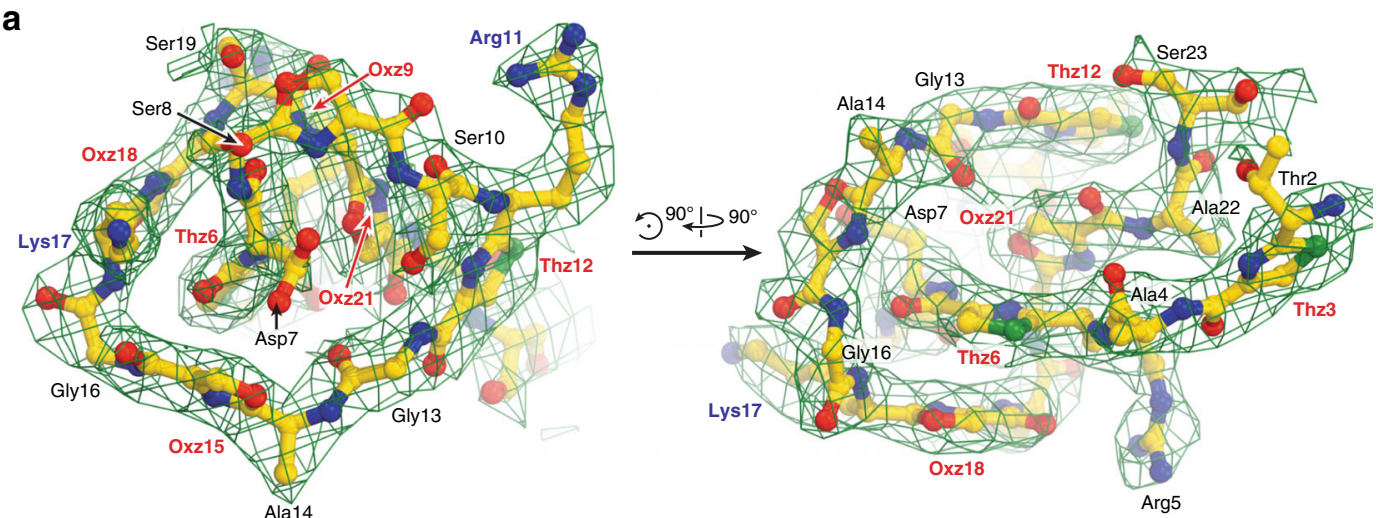

b

Phazolicin (PHZ)

Ala1 - Thr2 - Thz3 - Ala4 - Arg5 - Thz6 - Asp7 - Ser8 - Oxz9 - Ser10 - Arg11 - Thz12 - Gly13 - Ala14 - Oxz15 - Gly16 - Lys17 - Oxz18 - Ser19 - Gly20 - Oxz21 - Ala22 - Ser23 - Oxz24 - lle25 - Ser26 - Thr27

Observed in the charge density

C

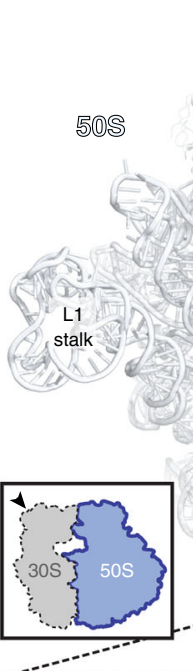

d

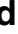

d
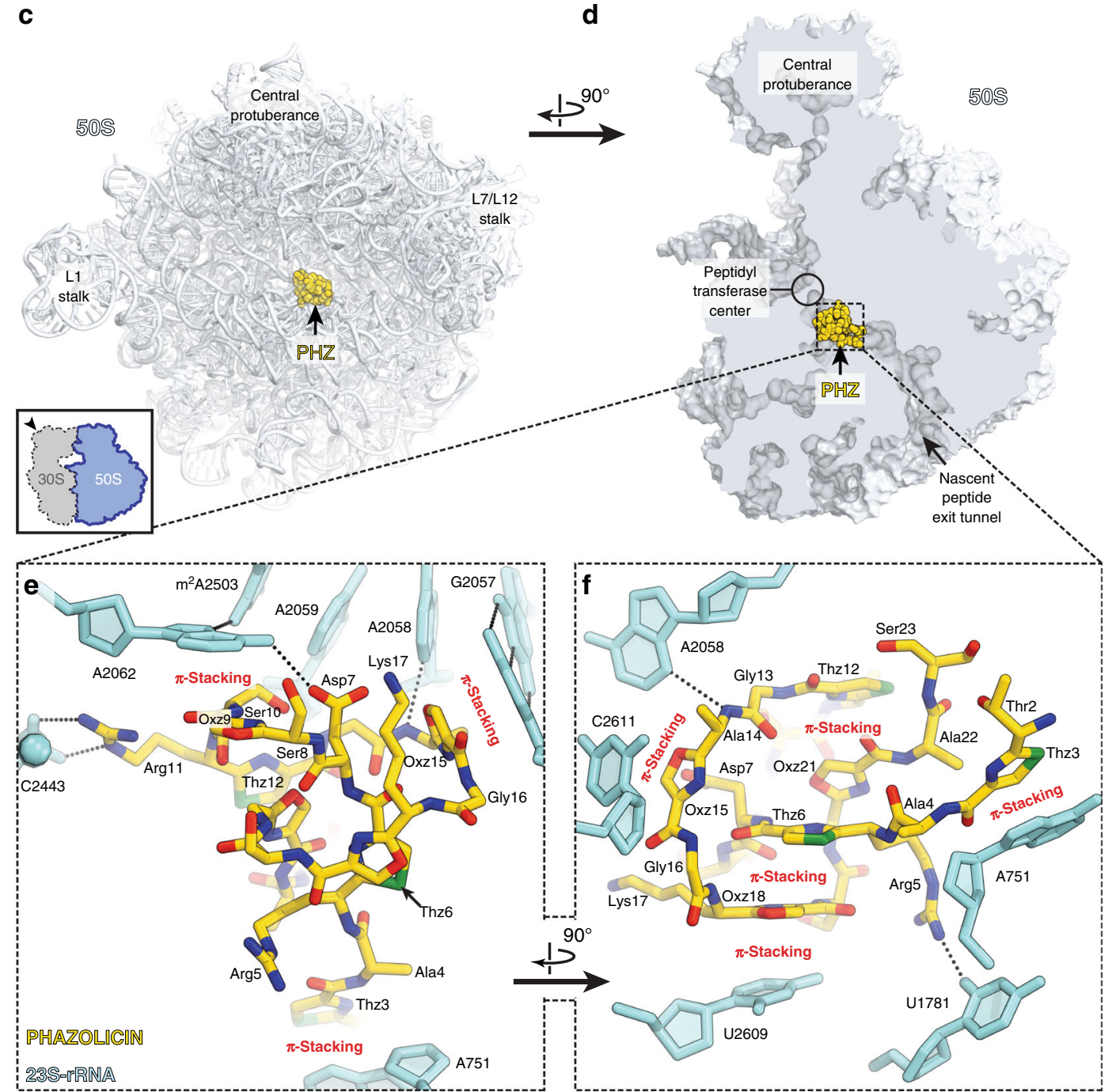

Fig. 3 The structure of $\mathrm{PHZ}$ in complex with the bacterial ribosome. a Cryo-EM map of PHZ in complex with the E. coli $70 S$ ribosome (green mesh). The fitted model of the compound is displayed in its respective charge density viewed from two different perspectives. The map is contoured at $2.5 \sigma$. Carbon atoms are colored yellow, nitrogen atoms are blue, oxygen atoms are red, and sulfur atoms are green. $\mathbf{b}$ Schematic diagram showing that only residues 2-23 of the ribosome-bound PHZ molecule are visible in the cryo-EM map. c, d Overview of the PHZ-binding site (yellow) on the E. coli large ribosomal subunit (light blue) viewed from two different perspectives. In c, the $50 \mathrm{~S}$ subunit is viewed from the inter-subunit interface (30S subunit is removed for clarity) as indicated by the inset. The view in $\mathbf{d}$ is from the cytoplasm onto the A site. $\mathbf{e}, \mathbf{f}$ Close-up views of the PHZ-binding site in the ribosome exit tunnel. E. coli numbering of the nucleotides in the $23 \mathrm{~S}$ rRNA is used. Potential $\mathrm{H}$-bond interactions are indicated with dotted lines 
was not visible in the map due to its poor quality in that region (Fig. 3a).

Similar to KLB, linear modified $\mathrm{PHZ}$ peptide forms a compact globule that binds in the upper part of the NPET of the 50S ribosomal subunit where it extensively interacts with the 23S rRNA and ribosomal proteins (Fig. 3c, d; Supplementary Movie 1). In comparison to antibiotics of smaller molecular weight, such as ERY, which binds at the same location but only partially occludes the NPET (Supplementary Fig. 6A, B), binding of both KLB and PHZ results in nearly complete obstruction of the NPET (Supplementary Fig. 6C, D). A prominent feature of the ribosome-bound $\mathrm{PHZ}$ molecule is its complex intramolecular interactions that involves both faceto-face and edge-to-face $\pi-\pi$ stacking of Thz12, Oxz21, Thz6, and Oxz18, along with the nucleobase U2609 from the 23S rRNA (Fig. 3f; Supplementary Fig. 7D). Moreover, we observed two major types of interactions between the PHZ and the nucleotides of the 23S rRNA. First, three azoles (Thz3, Oxz15, and Oxz18) are involved in $\pi-\pi$ stacking with nucleotides A751, C2611, and U2609, respectively (Fig. 3e, f). Second, two positively charged side chains of Arg5 and Arg11, as well as hydrophilic side chains of Asp7 and Ser8, form additional stabilizing hydrogen bonds (H-bonds) with nucleobases and backbone phosphate groups of the $23 \mathrm{~S}$ RNA (Fig. 3e, f; Supplementary Fig. 7A-C). The presence of intramolecular $\pi-\pi$ stacking and positively charged side chains, as well as the absence of the $\mathrm{N}$-terminal amidine cycle, make the mode of PHZ binding significantly different from that of KLB. Unlike $\mathrm{KLB}$, the $\mathrm{N}$-terminal part of $\mathrm{PHZ}$ is not critical for binding to the ribosome, which is further supported by the observation that naturally occurring longer forms with an alternative leader cleavage site (A-PHZ and TA-PHZ) demonstrate the same level of in vitro translation inhibition activity as PHZ (Supplementary Fig. 8).

Interestingly, the PHZ-binding site overlaps with those of several other classes of antibiotics, such as macrolides and type B streptogramins. Comparison of the structures of ribosome-bound PHZ and KLB reveals that PHZ binds further away than KLB from the peptidyl transferase center (PTC) of the ribosome. We superimposed our new structure of the ribosome-bound $\mathrm{PHZ}$ (Fig. 4a) or the previously published KLB (Fig. 4b) with the cryoEM structure of the $70 \mathrm{~S}$ ribosome containing ErmBL nascent peptide chain connected to the P-site tRNA ${ }^{31}$. Apparently, there is more space between the ribosome-bound drug and the PTC in the case of PHZ in comparison to KLB. This suggests that PHZ should, in principle, allow for a few more amino acids to be incorporated into the nascent polypeptide chain before it encounters the NPET-bound PHZ molecule and translation stalls. To test this hypothesis, we performed a toe-printing (primer-extension inhibition) assay, which allows identification of drug-induced ribosome stalling on a given mRNA template with a single-nucleotide precision ${ }^{32}$. Because PHZ causes strong induction (stalling of the ribosomes) on Dualrep2 reporter used in our in vivo bioactivity test (Fig. 2a), we have chosen the corresponding mRNA (trpL-2Ala) as a template for our toeprinting assay. Addition of $\mathrm{PHZ}$ to the PURExpress cell-free transcription-translation system programmed with $\operatorname{trpL-2Ala}$ mRNA resulted in dose-dependent ribosome stalling at the seventh codon of mRNA (Fig. 4c, lanes 4-7), while the addition of KLB induced major stalling at the third and the fifth codons (Fig. 4c, lane 3) corroborating our hypothesis. Moreover, these data indicate that PHZ is an inhibitor of the elongation step during protein synthesis, as is the KLB. In the absence of any inhibitors in our toe-printing reactions, ribosomes stall at codons 9-10 due to a specific secondary structure in the mRNA (Fig. 4c, lane 8).
Species-specificity of PHZ action. The PHZ-binding site is adjacent to the NPET constriction point-the most narrow part of the NPET formed by the extended loops of the ribosomal proteins uL4 and uL22 (Fig. 5a, b). The N-terminal and Cterminal parts of the PHZ molecule are located between the uL4 and uL22 loops (Fig. 5c) and occupy more space in this part of the NPET than the C-terminal part of KLB (Fig. 5d). The amino acid sequences of $\mathrm{uL} 4$ and $\mathrm{uL} 22$ proteins including their loop regions vary between distinct bacteria (Fig. 5e). Superpositioning of our Eco $70 \mathrm{~S}$ ribosome structure with bound $\mathrm{PHZ}$ with the structure of Tth $70 \mathrm{~S}$ reveals that residues His69 of the Tth protein uL4 sterically clashes with the PHZ molecule (Fig. 5c) rationalizing why PHZ does not bind to or act upon the Tth ribosome. Because the same residue in Eco ribosome is represented by a less bulky Gly64 (uL4), we hypothesized that the differences in the uL4 loop sequence are responsible for the inability of PHZ to bind in the NPET and, hence, to inhibit the translation by Tth ribosome. To test our hypothesis, we expressed a plasmid-borne copy of the $S$. meliloti rplD gene encoding for the uL4 protein carrying the Tth-like G68H substitution $\left(r p l D^{\mathrm{G} 68 \mathrm{H}}\right)$ and showed that the loop structure of Tth $\mathrm{uL} 4$ confers resistance to $\mathrm{PHZ}$ and allows growth of otherwise sensitive strain on the medium with 8xMIC of the antibiotic (Fig. 5f). The expression of $\mathrm{rplD}^{\mathrm{K} 65 \mathrm{~A}}$ mutant, which carries an alanine substitution of the Eco Arg61-equivalent (Fig. 5e) that forms $\mathrm{H}$-bond with the $\mathrm{PHZ}$ molecule in our structure (Fig. 5c), does not confer resistance to PHZ. This is likely due to multiple contacts, which the PHZ molecule forms with the ribosome, and the loss of one $\mathrm{H}$-bond does not affect the overall binding. The same superpositioning of the Eco and Tth ribosome structures also revealed that residue Arg90 of the Tth protein uL22 sterically clashes with the PHZ molecule (Fig. 5c), while the comparable in size-equivalent residue Lys90 in the Eco protein uL22 does not clash with the PHZ and is repositioned to the side relative to the ribosome-bound PHZ to avoid a similar clash. Unlike His69 $9^{\text {Tth }}$, which is located in a confined pocket and cannot re-adjust its position in response to $\mathrm{PHZ}$ binding, residues Arg90 Tth and Lys90 Eco have more space around and should be able to easily readjust their positions when PHZ is present to avoid a possible clash. Indeed, the substitution of Lys90 in the loop of protein $\mathrm{uL} 22$ with a larger Arg residue (rplV K90R) does not render any resistance to PHZ (Fig. 5f). Thus, the fine structure of the PHZ-binding site (formed in part by the loop regions of ribosomal proteins $\mathrm{uL} 4$ and $\mathrm{uL} 22$ ) together with the repertoire of peptide transporters involved in the uptake contribute to the specificity of the antibiotic action.

\section{Discussion}

The newly discovered antibiotic PHZ represents the second known example of a linear azole-containing peptide with a known mechanism of action and mode of binding to its intracellular target, the ribosome. Although the PHZ and the previously described LAP klebsazolicin both inhibit the elongation step of prokaryotic translation through the obstruction of the NPET of the large ribosomal subunit, the mode of binding of PHZ to the bacterial ribosome is significantly different from that of KLB. Unlike KLB, which does not appear to have extensive intramolecular interactions, four out of eight azole rings of PHZ form continuous $\pi-\pi$-stacking system (Supplementary Fig. 5D), which apparently confers the rigidity and stability of the antibiotic in its binding site. Moreover, PHZ contains several positively charged side chains, which provide additional anchoring on the ribosome through the electrostatic interactions with the negatively charged $23 \mathrm{~S}$ rRNA (Fig. 3e), and which were absent in KLB. Finally, PHZ appears to be the first ribosome-targeting antibiotic with a pronounced bacterial species-specific mode of 

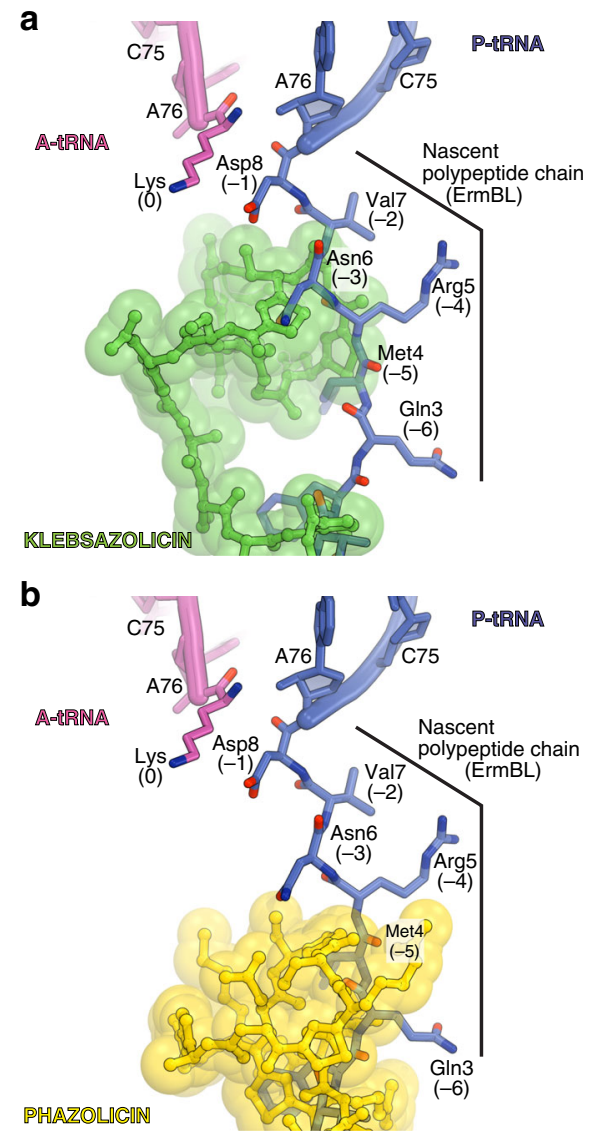

C

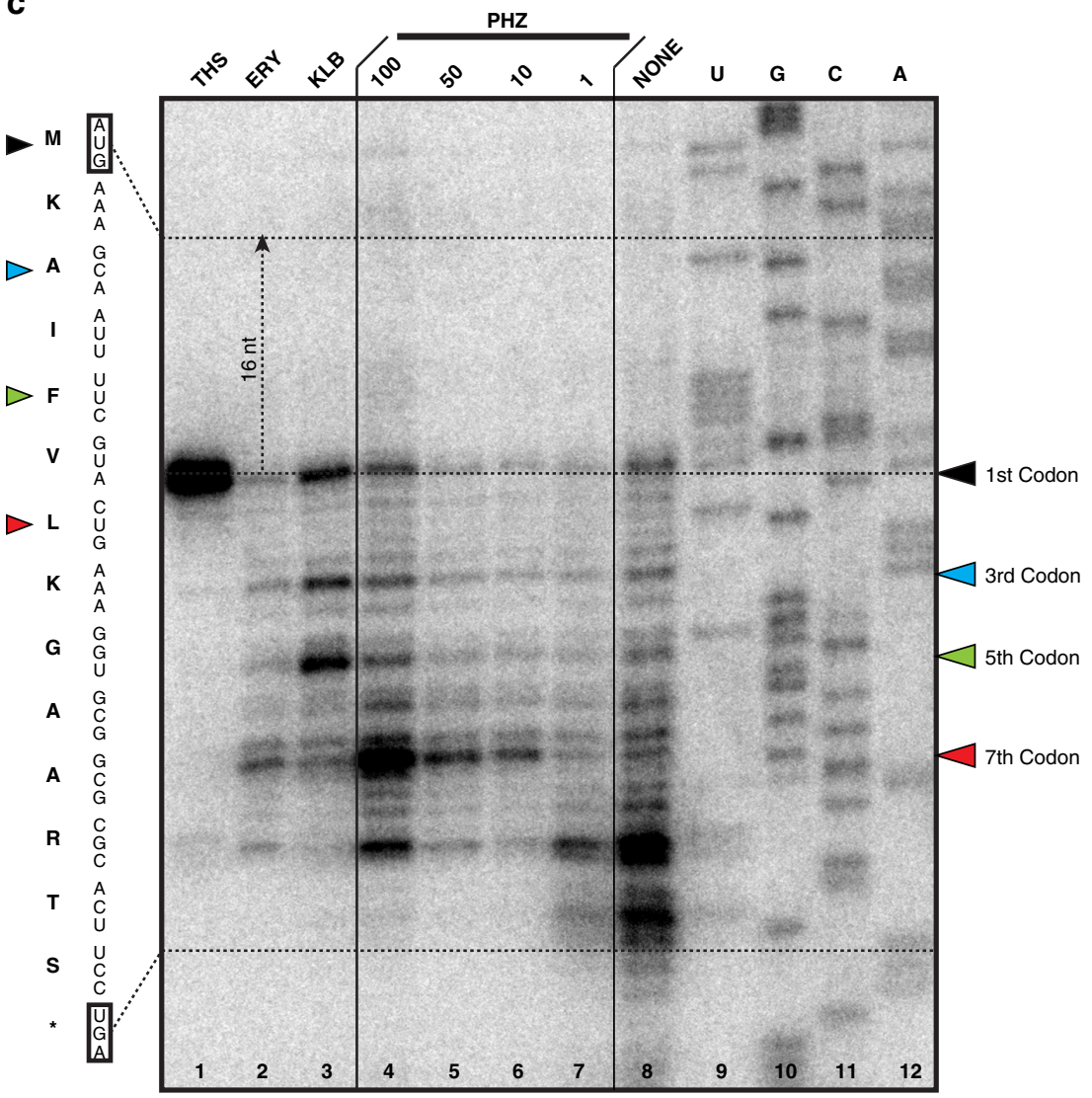

Fig. $4 \mathrm{PHZ}$ inhibits the elongation step during protein synthesis. $\mathbf{a}, \mathbf{b}$ In silico modeling of the nascent polypeptide chain in the ribosome exit tunnel in the presence of klebsazolicin ( $\mathbf{a}, \mathrm{PDB}$ entry $5 \mathrm{~W}_{4} \mathrm{~K}^{8}$ ) or phazolicin (b, current structure) using the cryo-EM structure of the 70S ribosome with ErmBL peptide bound to the P-site tRNA (PDB entry 5JTE ${ }^{31}$ ). c Ribosome stalling by PHZ on trpL-2Ala mRNA in comparison with other translation inhibitors (thiostrepton, THS; erythromycin, ERY; klebsazolicin, KLB), as revealed by reverse-transcription primer-extension inhibition (toe-printing) assay in a cell-free translation system. trpL-2Ala mRNA nucleotide sequence and the corresponding amino acid sequence are shown on the left. Colored triangles show ribosome stalling at $1 \mathrm{st}, 3 \mathrm{rd}$, 5th, and 7th codons. Note that owing to the large size of the ribosome, the reverse transcriptase stops at the nucleotide +16 relative to the codon located in the P-site

action, which is directly related to its mode of interaction with the molecular target. PHZ can bind to the ribosomes and act upon those species whose ribosomal protein uL4 contains glycine (or non-bulky side chain amino acid residues) in the position equivalent to Gly64 in E. coli that is located at the tip of the extended loop of this protein. The residue equivalent to Gly64 of the $E$. coli protein uL4 is not conserved in other bacterial species. The majority of bacteria (including E. coli) possess glycine at this position (Fig. 5e). However, others (e.g., T. thermophilus) contain histidine that renders them naturally resistant to this antibiotic due to a steric hindrance with PHZ molecule. There are many ribosome-targeting antibiotics that, as PHZ (see Table 1), work on one group of bacteria and do not work on the others, however, this phenomenon is usually not referred to as "species-specificity of action" because it is due to the ability of a drug to get inside the bacterial cell rather than a differential activity on its target.

To our knowledge, PHZ is the first example of an antibacterial species-specific ribosome-targeting inhibitor whose activity depends not only on its ability to penetrate inside the cell but also on the fine structure of its target, the bacterial $70 \mathrm{~S}$ ribosome (regardless of the methylation state of some of the nucleotides in the $23 \mathrm{~S}$ rRNA). Moreover, PHZ is the second known example of characterized RiPPs from the order Rhizobiales. Owing to the ability of nitrogen fixation and establishing symbiotic relationships with leguminous plants, rhizobia are considered as an agriculturally important group of bacteria that have been extensively studied for decades. However, even with dozens of full-genome sequences, the number of rhizobial natural products that were characterized to date remains extremely small in comparison to such fruitful groups as the Actinobacteria or the Enterobacteriales. At the same time, genome mining reveals significant potential for the discovery of other RiPPs in this group of bacteria. Of particular interest are the genes associated with the biosynthesis of LAPs and Nif11/NHLP-containing peptides ${ }^{33}$ present in multiple rhizobial genomes.

Characterization of PHZ together with the identification of BGCs of its predicted homologs in the genomes of other Rhizobiales expands the diversity of LAPs and illustrates how different RiPPs can share similar enzymatic machinery and, at the same time, can exhibit significantly different mechanisms of binding and action upon the target. The narrow-spectrum antibacterial activity of $\mathrm{PHZ}$ against rhizobia may serve as a starting point for the development of biocontrol agents, which could be used to combat for example plant-pathogenic Agrobacterium strains, or rhizobium inoculants for legume crops that can outcompete endogenous rhizobium strains.

\section{Methods}

Bacterial strains and growth conditions. The following bacterial strains were used in the present study: Rhizobium sp. Pop5, R. leguminosarum bv. viciae 3841, R. leguminosarum bv. phaseoli 4292, R. leguminosarum bv. phaseoli RCR 3622, $R$. etli DSM 11541, R. tibethicum DSM 21102, Agrobacterium tumefaciens C58C1, A. 
a

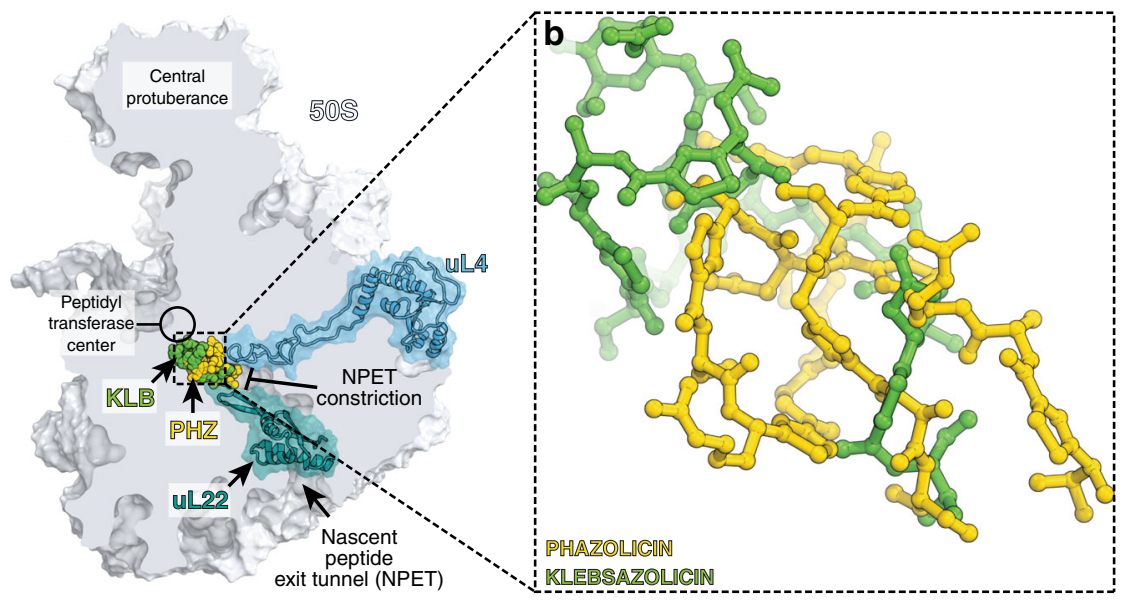

d

C

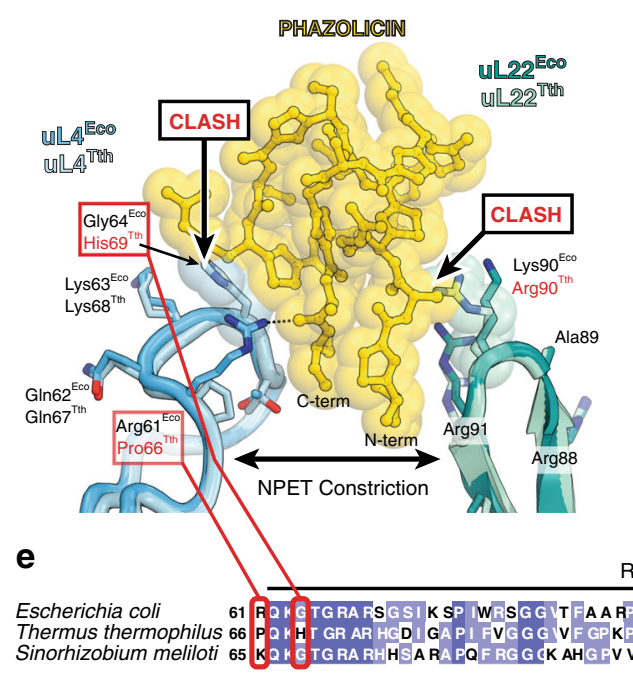

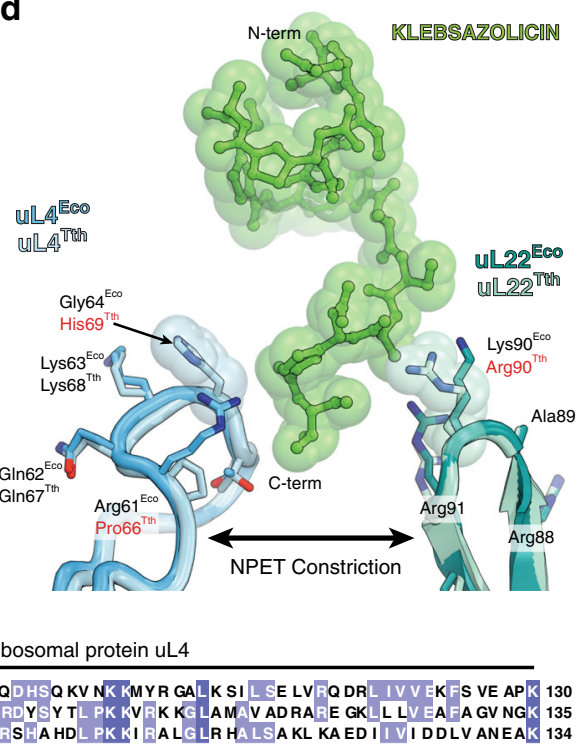

$\mathbf{f}$

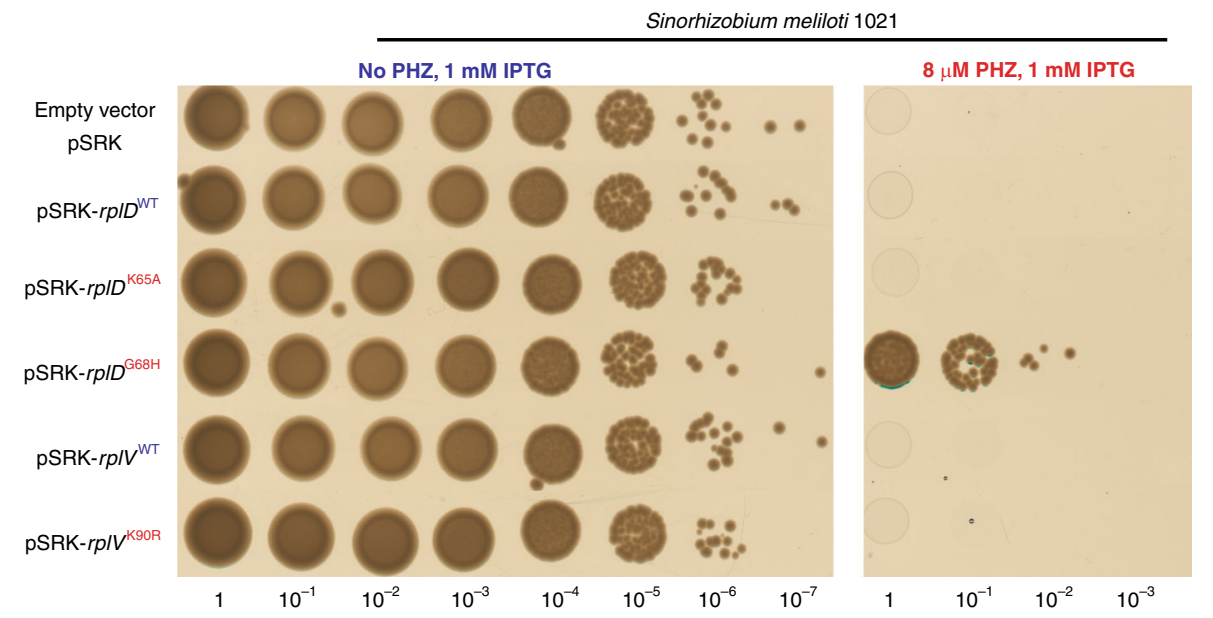

Fig. 5 The loop of ribosomal protein uL4 defines species-specificity of PHZ action. a Comparison of PHZ (yellow) and KLB (green) binding sites in the ribosome exit tunnel. The $50 S$ subunit is shown in light blue. Ribosomal proteins uL4 and uL22 are highlighted in blue and teal, respectively. b Superposition of the ribosome structures with bound KLB (PDB entry 5W4K ${ }^{8}$ ) and PHZ based on the alignment of the $23 \mathrm{~S}$ rRNAs. Ribosome parts are omitted for clarity. Note that KLB binds closer to the PTC than PHZ. c, d Close-up view of PHZ $\mathbf{c}$ and KLB $\mathbf{d}$ interactions with the loops of proteins uL4 and uL22 forming the NPET constriction site. Superposition of the loops of proteins from E. coli and T. thermophilus is shown. Note that sterical clashes occur only with the side chains in the loops of proteins uL4/uL22 from T. thermophilus. e Multiple sequence alignment of the homologous parts of the ribosomal protein uL4 from several bacterial species. Red boxes highlight the amino acid residues of uL4, which either clash with the PHZ (His69 in Tth) or interact with it in our structure (Arg61 in Eco). f Growth of S. meliloti Sm1021 and its derivatives expressing plasmid-borne copies of WT or mutant rpID or rpIV genes (encoding ribosomal proteins uL4 and uL22, respectively) on agar plates without PHZ (left panel) or with $8 \mu \mathrm{M} \mathrm{PHZ} \mathrm{(right} \mathrm{panel).} \mathrm{Note} \mathrm{that}$ only overexpression of Tth-like version of $\mathrm{uL} 4$ protein in S. meliloti confers resistance to $\mathrm{PHZ}$ 
rhizogenes ARQUA1, Sinorhizobium meliloti Sm1021, S. fredii HH103, S. medicae WSM419, Mesorhizobium ciceri, M. loti MAFF303099, M. thianshanense HAMBI 3372, Azorhizobium caulinodans ORS571, E. amylovora, P. ananatis PA4, P. putida KT2440, Pseudomonas fluorescens Pf-5, B. subtilis 168, Bacillus cereus ATCC 4342, Arthrobacter sp. ATCC21022, M. glauca NRRL B-3735, Escherichia coli DH5a, E. coli HB101, E. coli MRE600, E. coli BW25113, and E. coli BW25113 tolC. We used RM medium (per $1 \mathrm{~L}: 10 \mathrm{~g}$ mannitol, $0.5 \mathrm{~g} \mathrm{~K}_{2} \mathrm{HPO}_{4}, 0.2 \mathrm{~g} \mathrm{MgSO}_{4}, 0.1 \mathrm{~g} \mathrm{NaCl}, 1 \mathrm{~g}$ yeast extract, $\mathrm{pH}$ 6.8) for cultivation of various species of the Rhizobium genus (Table 1). Other rhizobia (including species of the genera Mesorhizobium, Sinorhizobium, Agrobacterium, and Azorhizobium) were cultivated in YEB medium (per $1 \mathrm{~L}: 5 \mathrm{~g}$ beef extract, $5 \mathrm{~g}$ peptone, $5 \mathrm{~g}$ sucrose, $1 \mathrm{~g}$ yeast extract, $0.2 \mathrm{~g} \mathrm{MgSO}_{4}, \mathrm{pH} 7.5$ ) (Table 1). All rhizobia strains were cultivated at $28^{\circ} \mathrm{C}$. Growth of all other strains was performed in $\mathrm{LB}$ medium (per $1 \mathrm{~L}: 5 \mathrm{~g} \mathrm{NaCl}, 10 \mathrm{~g}$ tryptone, and $5 \mathrm{~g}$ yeast extract) at 28 or $37^{\circ} \mathrm{C}$ (for E. coli strains). When necessary, antibiotics were used at concentrations of $100 \mu \mathrm{g} / \mathrm{ml}$ for kanamycin (for S. meliloti Sm1021), $50 \mu \mathrm{g} / \mathrm{ml}$ for kanamycin (for E. coli strains), $25 \mu \mathrm{g} / \mathrm{ml}$ for chloramphenicol, and $500 \mu \mathrm{g} / \mathrm{ml}$ for streptomycin.

Molecular cloning procedures. Primers used for DNA amplification are listed in Supplementary Table 1 . The $r p l D$ and $r p l V$ genes were amplified from Sinorhizobium meliloti Sm1021 genomic DNA using rplD_pSRK_GA_F/rplD_pSRK_GA_R and rplV_pSRK_GA_F/rplV_pSRK_GA_R pairs of primers, respectively, and cloned into the pSRK (Kan) broad-range host vector ${ }^{34}$ following the Gibson assembly protocol (NEB). Two mutant variants of the rplD gene and one mutant variant of $r p l V$ gene were obtained through PCR-based site-specific mutagenesis with primer pairs rplD_G68H_F/rplD_G68H_R, rplD_K65A_F/rplD_K65A_R, and rplV_K90R_F/rplV_K90R_R, and cloned into the pSRK plasmid as well. The phzE gene was amplified from Rhizobium sp. Pop5 genomic DNA using phzE_pSRK_GA_F and phzE_pSRK_GA R primers and cloned into pSRK. The transformation of Sinorhizobium meliloti Sm1021 with pRSK and pSRK-derived vectors was performed by triparental mating using pRK600 as a helper plasmid.

PHZ production and purification. Rhizobium sp. Pop5 was cultivated for $24 \mathrm{~h}$ in liquid $\mathrm{RM}$ medium $(750 \mathrm{ml})$ with shaking $(180 \mathrm{rpm})$ at $28^{\circ} \mathrm{C}$. Next, the cells were pelleted $\left(14,000 \mathrm{rpm}, 30 \mathrm{~min}, 4^{\circ} \mathrm{C}\right)$ and cultivation medium supplied with $0.1 \%$ trifluoroacetic acid (TFA) was loaded onto Agilent HF Bond Elut LRC-C18 Cartridge $(1 \mathrm{~g})$ pre-equilibrated with $0.1 \%$ TFA. The cartridge was then extensively washed with water followed by $50 \mathrm{ml}$ of $10 \%$ acetonitrile $(\mathrm{MeCN})$. The peptide fraction was eluted using $10 \mathrm{ml}$ of $30 \% \mathrm{MeCN}$, dried out with centrifugal evaporator (GeneVac EZ-2), and dissolved in $500 \mu \mathrm{l}$ of $100 \%$ dimethyl sulfoxide (DMSO). The obtained extract was then subjected to reverse phase HPLC purification on a Luna PREP C18 column $(21.2 \times 250 \mathrm{~mm}, 10 \mu \mathrm{m}$ particle size). The column was pre-equilibrated using $0.1 \%$ TFA (Buffer A). The following gradient of $100 \% \mathrm{MeCN}$ (Buffer B) was used for the separation and elution of the peptides: $0-5 \min 0 \%$ B, $5-12$ min $0-19 \%$ B, $12-22$ min $19-22 \%$ B, $22-27$ min $22-70 \%$ B, $27-3370 \%$ B. The detection was performed at $254 \mathrm{~nm}$. The collected fractions were then checked with MALDI-ToF MS and those containing the compounds of interest were dried under vacuum, dissolved in $100 \%$ DMSO and stored at $-20^{\circ} \mathrm{C}$ until further use.

High-resolution MS and ESI-MS-MS analysis. One microgram of peptides in a volume of $1-4 \mu \mathrm{L}$ was loaded onto the Acclaim $\mu$-Precolumn $(0.5 \mathrm{~mm} \times 3 \mathrm{~mm}$ $5 \mu \mathrm{m}$ particle size, Thermo Scientific) at a flow rate of $10 \mu \mathrm{L} / \mathrm{min}$ for $4 \mathrm{~min}$ in an isocratic mode of Mobile Phase C ( $2 \% \mathrm{MeCN}, 0.1 \%$ formic acid). Then the peptides were separated with high-performance liquid chromatography (HPLC, Ultimate 3000 Nano LC System, Thermo Scientific, Rockwell, IL, USA) in a 15-cm long C18 column (Acclaim ${ }^{\oplus}$ PepMap ${ }^{m}$ RSLC inner diameter of $75 \mu \mathrm{m}$, Thermo Fisher Scientific, Rockwell, IL, USA). The peptides were eluted with a gradient of buffer B $(80 \% \mathrm{MeCN}, 0.1 \%$ formic acid) at a flow rate of $0.3 \mu \mathrm{l} / \mathrm{min}$. The total run time was $60 \mathrm{~min}$, which included an initial $4 \mathrm{~min}$ of column equilibration with buffer A ( $0.1 \%$ formic acid), then gradient from $5 \%$ to $35 \%$ of buffer B over $35 \mathrm{~min}$, then 6 min to reach $99 \%$ of buffer B, flushing 10 min with $99 \%$ of buffer B and 5 min reequilibration with buffer $A$.

MS analysis was performed at least in triplicate with a Q Exactive HF mass spectrometer (Q Exactive HF Hybrid Quadrupole-OrbitrapTM Mass spectrometer, Thermo Fisher Scientific, Rockwell, IL, USA). The temperature of the capillary was $240{ }^{\circ} \mathrm{C}$ and the voltage at the emitter was $2.1 \mathrm{kV}$. Mass spectra were acquired at a resolution of 120,000 (MS) in a range of $300-1500 \mathrm{~m} / \mathrm{z}$. Tandem mass spectra of fragments were acquired at a resolution of 15,000 (MS/MS) in the range from $100 \mathrm{~m} / \mathrm{z}$ to $1200 \mathrm{~m} / z$ value determined by a charge state of the precursor, but no more than $2000 \mathrm{~m} / z$. The maximum integration time was 50 and $110 \mathrm{~ms}$ for precursor and fragment ions, respectively. Automatic gain control (AGC) target for precursor and fragment ions were set to $1^{\star} 10^{6}$ and $2^{\star} 10^{5}$, respectively. An isolation intensity threshold of 50,000 counts was determined for precursor's selection and up to top 20 precursors were chosen for fragmentation with high-energy collisional dissociation (HCD) at 29 normalized collision energy (NCE). Precursors with a charge state of +1 and more than +5 were rejected and all measured precursors were dynamically excluded from triggering of a subsequent MS/MS for $20 \mathrm{~s}$.
MALDI-ToF-MS and MS-MS analysis. MALDI-MS mass spectra were recorded in a positive ion measurement mode using Ultraflextreme MALDI-ToF-ToF-MS instrument (Bruker Daltonics, Germany). The spectra were obtained in reflectomode with the accuracy of measuring the monoisotopic $\mathrm{m} / \mathrm{z}$ ratio up to $0.1 \mathrm{Da}$. The fragmentation spectra were obtained using the Lift mode, for which the accuracy of measuring the daughter ions was within $0.2 \mathrm{Da}$. Sample aliquots were mixed on a steel target with a $30 \mathrm{mg} / \mathrm{ml} 2,5$-dihydroxybenzoic acid in $0.5 \%$ TFA and a $30 \%$ $\mathrm{MeCN}$-water solution (Sigma-Aldrich) (see Supplementary Data 1-4).

Determination of MICs. Determination of MICs was performed in 96-well plates in different media for different bacterial strains (Table 1) using a microdilution assay. In each row, the next well contained two times less antibiotic than the previous one. Two different concentration ranges were tested: $128-0.5 \mu \mathrm{M}$ for the strains less susceptible to PHZ; and $8 \mu \mathrm{M}$ to $31.25 \mathrm{nM}$ for the strains more susceptible to PHZ; a well without antibiotic was used as a control. Initially each well contained $100 \mu \mathrm{l}$ of media. Actively growing bacteria were added to the wells up to the concentration of $\sim 5^{\star} 10^{6}$ cells $/ \mathrm{mL}$. The plates were incubated for $48 \mathrm{~h}$ at $28^{\circ} \mathrm{C}$ with shaking $(180 \mathrm{rpm})$. MIC was defined as the concentration of PHZ in the well, in which no bacterial growth was observed, while the two-fold lower concentration of PHZ was not abolishing the growth of bacteria.

Testing of PHZ activity in vivo. For the in vivo bioactivity test (Fig. 2a), the reporter strain BW25113-pDualrep2 and BW25113 ttolC-pDualrep2 was used as previously described ${ }^{19}$. Briefly, $2 \mu \mathrm{L}$ of solutions of tested compounds were applied onto the agar plate that already contained a lawn of the reporter strain. $10 \mathrm{mM}$ PHZ, KLB, ERY, and $20 \mu \mathrm{M}$ levofloxacin solutions were used. After overnight incubation of the plate at $37^{\circ} \mathrm{C}$, it was scanned by ChemiDoc (Bio-Rad) using "Cy3-blot" settings for RFP and "Cy5-blot" for the Katushka2S protein.

In vitro translation inhibition assays. The inhibition of firefly luciferase synthesis by PHZ (Fig. 2b) was assessed essentially as described previously ${ }^{23}$. Briefly, the in vitro transcribed firefly luciferase mRNA was translated in the E. coli S30 Extract System for Linear Templates (Promega). Reactions containing $200 \mathrm{ng}$ of mRNA and $0.1 \mathrm{mM}$ of $\mathrm{D}$-luciferin were carried out in $5 \mu \mathrm{L}$ aliquots at $37^{\circ} \mathrm{C}$ for $15 \mathrm{~min}$ and the activity of in vitro synthesized luciferase was measured by VictorX5 (PerkinElmer) every $30 \mathrm{~s}$

To test the ability of PHZ to act upon the E. coli vs. T. thermophilus ribosomes (Supplementary Fig. 3), we used in vitro PURExpress translation system (New England Biolabs) reconstituted from purified components and supplied with either the WT 70S ribosomes from E. coli or the reconstituted hybrid 70S ribosomes containing purified $30 \mathrm{~S}$ subunits from E. coli and purified 50S subunits from $T$. thermophilus. Translation of superfolder green fluorescent protein (sfGFP) was carried out according to the manufacturer's protocol. The assembled reactions $(10 \mu \mathrm{l})$ were supplemented with $100 \mathrm{ng}$ of sfGFP mRNA. PHZ, KLB, or CHL (chloramphenicol) antibiotics we added to a final concentration of $50 \mu \mathrm{M}$ when needed. The reactions were placed in a 384-well black-wall plate and the progression of the reactions was monitored over $3 \mathrm{~h}$ by a TECAN microplate scanner.

Toe-printing analysis. Toe-printing analysis was carried out essentially as previously described ${ }^{35}$, using trpL-2Ala mRNA as a template for protein translation. trpL-2Ala template was prepared by PCR from pDualrep2 plasmid with following oligonucleotides: F TGTAATACGACTCACTATAGGGAATTTTTCTGTATAAT AGCCGCGGAAGTTCACGTAAAAAGGGTATCGACAATGAA and R GCGTT AAGGCTATGTACGGGTATCTGATTGCTTTACG, GCGTTAAGGCTATGTAC oligonucleotide was used for reverse transcription. The concentrations of the tested compounds are shown above each lane in Fig. 2e.

Cryo-EM structure determination. Ribosome preparation: E. coli strain MRE600 overnight cultures were diluted 1:100 into $3 \mathrm{~L}$ of $\mathrm{LB}$. After growth to mid-log phase $\left(\mathrm{OD}_{600}=0.6\right)$, cells were cooled on ice for $3 \mathrm{~min}$, pelleted, and washed with buffer A (20 mM Tris- $\mathrm{HCl} \mathrm{pH} \mathrm{7.5,} 100 \mathrm{mM} \mathrm{NH}_{4} \mathrm{Cl}, 10 \mathrm{mM} \mathrm{MgCl} 2,0.5 \mathrm{mM}$ EDTA, $2 \mathrm{mM}$ DTT). Cells were resuspended in $100 \mathrm{ml}$ buffer AS (buffer A with $0.15 \mathrm{M}$ sucrose) and lysed with two passes through an Emulsiflex-C3 homogenizer at 18,000 psi. Lysate was clarified by centrifugation for $30 \mathrm{~min}$ at $18,000 \mathrm{rpm}$ in a JA-20 rotor (Beckman-Coulter). The supernatant was layered over a sucrose cushion of $24 \mathrm{ml}$ buffer B (buffer A with $500 \mathrm{mM} \mathrm{NH}_{4} \mathrm{Cl}$ ) with $0.5 \mathrm{M}$ sucrose and $17 \mathrm{ml}$ buffer $\mathrm{C}$ (20 mM Tris- $\mathrm{HCl}$ pH 7.5, $60 \mathrm{mM} \mathrm{NH}_{4} \mathrm{Cl}, 6 \mathrm{mM} \mathrm{MgCl}_{2}, 0.5 \mathrm{mM}$ EDTA, $2 \mathrm{mM}$ DTT) with $0.7 \mathrm{M}$ sucrose in ti-45 tubes (Beckman-Coulter). Ribosomes were pelleted in a Ti- 45 rotor at $27,000 \mathrm{rpm}$ for $16 \mathrm{~h}$ at $4{ }^{\circ} \mathrm{C}$. Crude ribosome pellets were resuspended in disassociation buffer (buffer $\mathrm{C}$ with $1 \mathrm{mM} \mathrm{MgCl}$ ) and clarified via centrifugation at $15,000 \times g$ for $10 \mathrm{~min}$. The cleared supernatant was layered over $15-40 \%$ sucrose gradients in disassociation buffer and spun $28,000 \mathrm{rpm}$ for $16 \mathrm{~h}$ at $4{ }^{\circ} \mathrm{C}$ in a SW-32 rotor (Beckman-Coulter). Gradients were fractionated with an ISCO fractionation system and $30 \mathrm{~S}+50 \mathrm{~S}$ peaks were combined. The combined subunits were concentrated in $15 \mathrm{ml}$ Millipore 100k molecular weight cutoff spin filter and buffer exchanged with reassociation buffer (buffer $\mathrm{C}$ with $10 \mathrm{mM} \mathrm{MgCl}_{2}$ ). Ribosomes were incubated $45 \mathrm{~min}$ at $37^{\circ} \mathrm{C}$, layered onto $15-40 \%$ sucrose gradients in reassociation buffer, and spun $27,000 \mathrm{rpm}$ for $15 \mathrm{~h}$ at $4^{\circ} \mathrm{C}$ in an SW-32 rotor. 
Gradients were fractionated as before, 70 S peaks were collected, and ribosomes were concentrated and washed with buffer $\mathrm{C}$ in an Amicon stirred cell filtration system using 100k cutoff filters. Ribosomes were stored in aliquots at $-80^{\circ} \mathrm{C}$.

Cryo-EM sample preparation: Roughly 4-6 $\mu \mathrm{M}$ PHZ was incubated with $100 \mathrm{nM}$ ribosomes in RC buffer (50 mM Hepes pH 7.5, $150 \mathrm{mM} \mathrm{KOAc,} 12 \mathrm{mM}$ $\mathrm{MgOAc}, 7 \mathrm{mM} \beta$-mercaptoethanol) for $30 \mathrm{~min}$. at $37^{\circ} \mathrm{C}$. Complexes were deposited in $4-\mu \mathrm{l}$ aliquots on 300 mesh Quantifoil UltraAuFoil R1.2/1.3 grids that were topped with a layer of amorphous continuous carbon and glow-discharged for $15 \mathrm{~s}$ with a Pelco SC-6 sputter coater. After incubation period of about a minute, the excess sample was washed off on drops of RC-LS buffer (RC with $25 \mathrm{mM}$ $\mathrm{KOAc}$ ). Samples were blotted and plunge-frozen in liquid ethane using an FEI Vitrobot with blot force 6 , humidity $100 \%$, and 20 or $4{ }^{\circ} \mathrm{C}$ depending on the freezing session.

Data collection: Images were collected in two separate sessions on an FEI Titan Krios Microscope operated at $300 \mathrm{keV}$ with a GIF energy filter and GATAN Summit K2 direct electron detector in super-resolution mode. Images were collected at $\times 215,000$ magnification for a pixel size of $0.56 \AA(0.28 \AA$ superresolution). Automated movie collection was performed with SerialEM ${ }^{36}$ over the defocus range -0.6 to $-2.0 \mu \mathrm{m}$, and Focus software ${ }^{37}$ was used to monitor data collection. The total dose was $29.97 \mathrm{e}^{-} / \AA^{2}$ for session 1 and $29.83 \mathrm{e}^{-} / \AA^{2}$ for session 2, each over 30 frames.

Data processing: Cryo-EM data processing was done using RELION 3.0 software $^{38}$ (Supplementary Fig. 9; Supplementary Table 2). The two datasets were processed separately until the final round of map refinement, for which the best particles from both sets were combined. Movies were motion-corrected and dose-weighted using RELION's motion correction algorithm. Contrast transfer function (CTF) parameters were estimated using CTFFind $4^{39}$ and micrographs with poor CTF fit, as determined by visual inspection, were sorted out. Particles were picked automatically with the Laplacian-of-Gaussian method and subjected to several rounds of careful 2D class-based cleaning before an initial round of 3D auto-refine. CTF refinement with per-particle defocus and beam tilt correction was performed, and particles were further sorted through 3D classification without alignment. Another round of 3D auto-refine was performed on the best 3D classes with focused refinement on the 50S subunit. This was followed by one round of Bayesian polishing ${ }^{38}$ and another round of CTF refinement, which were each followed by another round of 3D auto-refine with a mask around the 50S subunit. Particles from the two sessions were then pooled for the final round of 3D autorefine. A charge density map was calculated in Chimera ${ }^{40}$ as previously described ${ }^{41}$. Post-processing was also performed in RELION to generate a FSC curve. All refinement procedures were run on $2 \times$ binned particles because of the small pixel size.

Model building: Assembly \#2 from the PDB entry $4 \mathrm{YBB}^{42}$ was used as a starting ribosome model. Only the 50S subunit was subjected to real-space refinement in PHENIX ${ }^{43}$, because charge density corresponding to the 30 S subunit was relatively poor after focused refinement on the 50S, where PHZ binds. Ions and water molecules were removed from the starting model prior to real-space refinement. The atomic model for the PHZ was built as follows: first, baton building in $\mathrm{COOT}^{44}$ was used to trace the main chain; next, the resulting polyalanine chain was mutated to the unmodified PHZ sequence after visual search for the characteristic features in the map; next, oxazole and thiazole modifications were built in Avogadro ${ }^{45}$ version 1.2.0 (http://avogadro.cc) based on the density. Finally, CIF restraints for the oxazole and thiazole moieties were created based on the structural data from ref. ${ }^{4}$.

All figures showing atomic models were generated using the PyMol software (www.pymol.org). USCF Chimera was used for EM density maps shown in Figs. S5 and S9.

Reporting summary. Further information on research design is available in the Nature Research Reporting Summary linked to this article.

\section{Data availability}

The data that support the findings of this study are available from the corresponding author upon request. Coordinates and charge density map were deposited in the RCSB Protein Data Bank with the accession code 6U48 for the E. coli 50S ribosomal subunit in complex with PHZ. The mass-spectrometric data presented in Supplementary Figs. 1C, 2, $3 \mathrm{~A}, \mathrm{~B}$ are provided as a Supplementary Data file.

Received: 13 July 2019; Accepted: 9 September 2019; Published online: 08 October 2019

\section{References}

1. Newman, D. J. \& Cragg, G. M. Natural products as sources of new drugs from 1981 to 2014. J. Nat. Prod. 79, 629-661 (2016).

2. Rossiter, S. E., Fletcher, M. H. \& Wuest, W. M. Natural products as platforms to overcome antibiotic resistance. Chem. Rev. 117, 12415-12474 (2017).
3. Skinnider, M. A. et al. Genomic charting of ribosomally synthesized natural product chemical space facilitates targeted mining. Proc. Natl Acad. Sci. USA 113, E6343-E6351 (2016).

4. Arnison, P. G. et al. Ribosomally synthesized and post-translationally modified peptide natural products: overview and recommendations for a universal nomenclature. Nat. Prod. Rep. 30, 108-160 (2013).

5. Burkhart, B. J., Schwalen, C. J., Mann, G., Naismith, J. H. \& Mitchell, D. A. YcaO-dependent posttranslational amide activation: biosynthesis, structure, and function. Chem. Rev. 117, 5389-5456 (2017).

6. Pierrat, O. A. \& Maxwell, A. The action of the bacterial toxin microcin B17. Insight into the cleavage-religation reaction of DNA gyrase. J. Biol. Chem. 278, 35016-35023 (2003).

7. Molohon, K. J. et al. Plantazolicin is an ultra-narrow spectrum antibiotic that targets the Bacillus anthracis membrane. ACS Infect. Dis. 2, 207-220 (2016).

8. Metelev, M. et al. Klebsazolicin inhibits $70 \mathrm{~S}$ ribosome by obstructing the peptide exit tunnel. Nat. Chem. Biol. 13, 1129-1136 (2017).

9. Roslycky, E. B. Bacteriocin production in the rhizobia bacteria. Can. J. Microbiol. 13, 431-432 (1967).

10. Triplett, E. W. \& Barta, T. M. Trifolitoxin production and nodulation are necessary for the expression of superior nodulation competitiveness by Rhizobium leguminosarum bv. trifolii strain T24 on clover. Plant. Physiol. 85, 335-342 (1987).

11. Altschul, S. F. et al. Gapped BLAST and PSI-BLAST: a new generation of protein database search programs. Nucleic Acids Res. 25, 3389-3402 (1997).

12. Travin, D. Y. et al. Biosynthesis of translation inhibitor klebsazolicin proceeds through heterocyclization and $\mathrm{N}$-terminal amidine formation catalyzed by a single YcaO enzyme. J. Am. Chem. Soc. 140, 5625-5633 (2018).

13. Metelev, M., Serebryakova, M., Ghilarov, D., Zhao, Y. \& Severinov, K. Structure of microcin B-like compounds produced by Pseudomonas syringae and species specificity of their antibacterial action. J. Bacteriol. 195, 4129-4137 (2013).

14. Letzel, A. C., Pidot, S. J. \& Hertweck, C. Genome mining for ribosomally synthesized and post-translationally modified peptides (RiPPs) in anaerobic bacteria. BMC Genom. 15, 983 (2014).

15. Mills, S., Ross, R. P. \& Hill, C. Bacteriocins and bacteriophage; a narrowminded approach to food and gut microbiology. FEMS Microbiol. Rev. 41, S129-S153 (2017).

16. Severi, E. \& Thomas, G. H. Antibiotic export: transporters involved in the final step of natural product production. Microbiology 165, 805-818 (2019).

17. Maksimov, M. O. \& Link, A. J. Discovery and characterization of an isopeptidase that linearizes lasso peptides. J. Am. Chem. Soc. 135, 12038-12047 (2013)

18. Tikhonov, A. et al. The mechanism of microcin $\mathrm{C}$ resistance provided by the MccF peptidase. J. Biol. Chem. 285, 37944-37952 (2010).

19. Osterman, I. A. et al. Sorting out antibiotics' mechanisms of action: a double fluorescent protein reporter for high throughput screening of ribosome and DNA biosynthesis inhibitors. Antimicrob. Agents Chemother. 60, 7481-7489 (2016).

20. Bleuel, C. et al. TolC is involved in enterobactin efflux across the outer membrane of Escherichia coli. J. Bacteriol. 187, 6701-6707 (2005).

21. Kobayashi, N., Nishino, K. \& Yamaguchi, A. Novel macrolide-specific ABCtype efflux transporter in Escherichia coli. J. Bacteriol. 183, 5639-5644 (2001).

22. Delgado, M. A., Vincent, P. A., Farias, R. N. \& Salomon, R. A. YojI of Escherichia coli functions as a microcin J25 efflux pump. J. Bacteriol. 187, 3465-3470 (2005)

23. Polikanov, Y. S. et al. Amicoumacin A inhibits translation by stabilizing mRNA interaction with the ribosome. Mol. Cell 56, 531-540 (2014).

24. Almutairi, M. M. et al. Co-produced natural ketolides methymycin and pikromycin inhibit bacterial growth by preventing synthesis of a limited number of proteins. Nucleic Acids Res. 45, 9573-9582 (2017).

25. Osterman, I. A. et al. Madumycin II inhibits peptide bond formation by forcing the peptidyl transferase center into an inactive state. Nucleic Acids Res. 45, 7507-7514 (2017).

26. Khabibullina, N. F. et al. Structure of dirithromycin bound to the bacterial ribosome suggests new ways for rational improvement of macrolides. Antimicrob. Agents Chemother. 63, e02266-18 (2019).

27. Svetlov, M. S. et al. High-resolution crystal structures of ribosome-bound chloramphenicol and erythromycin provide the ultimate basis for their competition. RNA 25, 600-606 (2019).

28. Tereshchenkov, A. G. et al. Binding and action of amino acid analogs of chloramphenicol upon the bacterial ribosome. J. Mol. Biol. 430, 842-852 (2018).

29. Polikanov, Y. S. et al. Distinct trna accommodation intermediates observed on the ribosome with the antibiotics hygromycin A and A201A. Mol. Cell 58, 832-844 (2015).

30. Pantel, L. et al. Odilorhabdins, antibacterial agents that cause miscoding by binding at a new ribosomal site. Mol. Cell 70, 83-94 (2018). 
31. Arenz, S. et al. A combined cryo-EM and molecular dynamics approach reveals the mechanism of ErmBL-mediated translation arrest. Nat. Commun. 7, 12026 (2016).

32. Orelle, C. et al. Tools for characterizing bacterial protein synthesis inhibitors. Antimicrob. Agents Chemother. 57, 5994-6004 (2013).

33. Haft, D. H., Basu, M. K. \& Mitchell, D. A. Expansion of ribosomally produced natural products: a nitrile hydratase- and Nif11-related precursor family. BMC Biol. 8, 70 (2010).

34. Khan, S. R., Gaines, J., Roop, R. M. 2nd \& Farrand, S. K. Broad-host-range expression vectors with tightly regulated promoters and their use to examine the influence of TraR and TraM expression on Ti plasmid quorum sensing. Appl. Environ. Microbiol. 74, 5053-5062 (2008).

35. Orelle, C. et al. Identifying the targets of aminoacyl-tRNA synthetase inhibitors by primer extension inhibition. Nucleic Acids Res. 41, e144 (2013).

36. Mastronarde, D. N. Automated electron microscope tomography using robust prediction of specimen movements. J. Struct. Biol. 152, 36-51 (2005).

37. Biyani, N. et al. Focus: the interface between data collection and data processing in cryo-EM. J. Struct. Biol. 198, 124-133 (2017).

38. Zivanov, J. et al. New tools for automated high-resolution cryo-EM structure determination in RELION-3. Elife 7, e42166 (2018).

39. Rohou, A. \& Grigorieff, N. CTFFIND4: fast and accurate defocus estimation from electron micrographs. J. Struct. Biol. 192, 216-221 (2015).

40. Pettersen, E. F. et al. UCSF Chimera-a visualization system for exploratory research and analysis. J. Comput. Chem. 25, 1605-1612 (2004).

41. Wang, J. Experimental charge density from electron microscopic maps. Protein Sci. 26, 1619-1626 (2017).

42. Noeske, J. et al. High-resolution structure of the Escherichia coli ribosome. Nat. Struct. Mol. Biol. 22, 336-341 (2015).

43. Adams, P. D. et al. PHENIX: a comprehensive Python-based system for macromolecular structure solution. Acta Crystallogr. D 66, 213-221 (2010).

44. Emsley, P., Lohkamp, B., Scott, W. G. \& Cowtan, K. Features and development of Coot. Acta Crystallogr. D 66, 486-501 (2010).

45. Hanwell, M. D. et al. Avogadro: an advanced semantic chemical editor, visualization, and analysis platform. J. Cheminform. 4, 17 (2012).

46. You, S.-L. \& Kelly, J. W. The total synthesis of bistratamides F-I. Tetrahedron 61, 241-249 (2005).

\section{Acknowledgements}

We thank Dr. Viktor Zgoda for the invaluable help with high-resolution spectra and ESI-MS-MS spectra acquisition and Dr. Esperanza Martinez-Romero who provided the strain Rhizobium sp. Pop5. We thank all members of the K.S., J.H.D.C., and Y.S.P. laboratories for important discussions and critical feedback. We also want to thank Dr. Dmitry Ghilarov and Dr. Svetlana Dubiley for critical reading of the manuscript and valuable suggestions. This work was supported by RSF grant number 19-14-00266 to Svetlana Dubiley, RSF grant number 18-44-04005 to I.A.O. (used for the toe-printing assays and in vivo (pDualrep2 reporter) and in vitro activity tests), by Illinois State startup funds [to Y.S.P.], National Institutes of Health [R01-GM132302 and R21AI137584 to Y.S.P., R01-GM114454 to J.H.D.C. and F.R.W.] and by the National Science
Foundation Graduate Research Fellowship Program under Grant number 1106400 [to Z.L.W.]. Access to MALDI-MS facilities was through the framework of the Lomonosov Moscow State University Development Program PNR 5.13.

\section{Author contributions}

D.Y.T. and M.M. performed genome mining, isolation, and purification of the compound; D.Y.T., M.M., I.A.O., I.M.K., P.M., and N.F.K. evaluated the bioactivity of PHZ and performed biochemical assays; Z.L.W., F.R.W., and J.H.D.C performed cryo-EM structural studies of $\mathrm{PHZ}$ in complex with E. coli ribosome and analyzed results with the input from Y.S.P.; M.S. and D.Y.T. performed MS experiments and analyzed data; D.Y.T. designed and performed genetic experiments showing species-specific mode of PHZ action. All authors interpreted the results. D.Y.T., Y.S.P., J.H.D.C., and K.S. wrote the manuscript.

\section{Competing interests}

The authors declare no competing interests.

\section{Additional information}

Supplementary information is available for this paper at https://doi.org/10.1038/s41467019-12589-5.

Correspondence and requests for materials should be addressed to Y.S.P., J.H.D.C. or K.S.

Peer review information Nature Communications thanks Matthieu Gagnon, and the other, anonymous, reviewer(s) for their contribution to the peer review of this work. Peer reviewer reports are available.

Reprints and permission information is available at http://www.nature.com/reprints Publisher's note Springer Nature remains neutral with regard to jurisdictional claims in published maps and institutional affiliations.

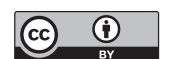

Open Access This article is licensed under a Creative Commons Attribution 4.0 International License, which permits use, sharing, adaptation, distribution and reproduction in any medium or format, as long as you give appropriate credit to the original author(s) and the source, provide a link to the Creative Commons license, and indicate if changes were made. The images or other third party material in this article are included in the article's Creative Commons license, unless indicated otherwise in a credit line to the material. If material is not included in the article's Creative Commons license and your intended use is not permitted by statutory regulation or exceeds the permitted use, you will need to obtain permission directly from the copyright holder. To view a copy of this license, visit http://creativecommons.org/ licenses/by/4.0/.

(c) The Author(s) 2019 